PLASMA PHYSICS OROUF

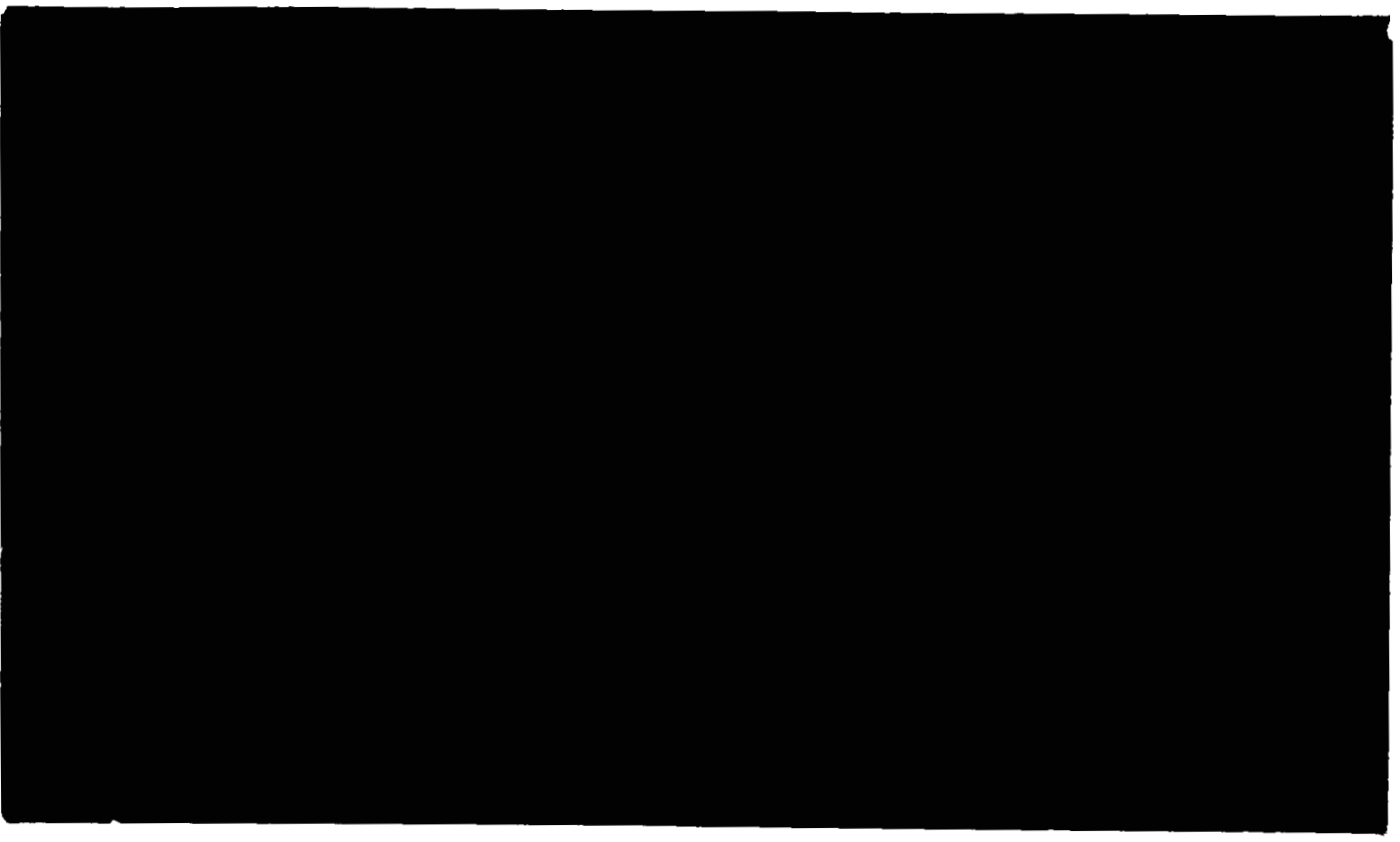

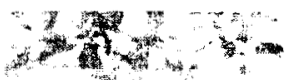

MYODEARTMENT OF RHYESES 13.

, o

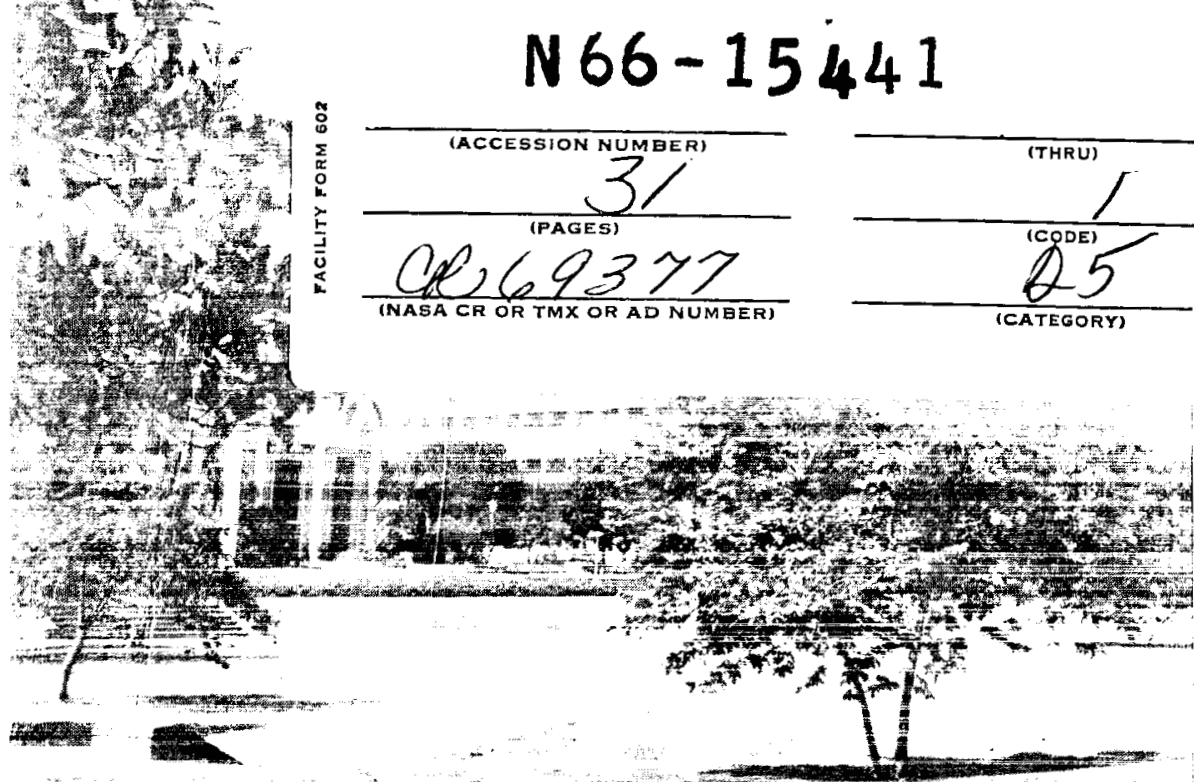

N $66-15441$

GPO F :
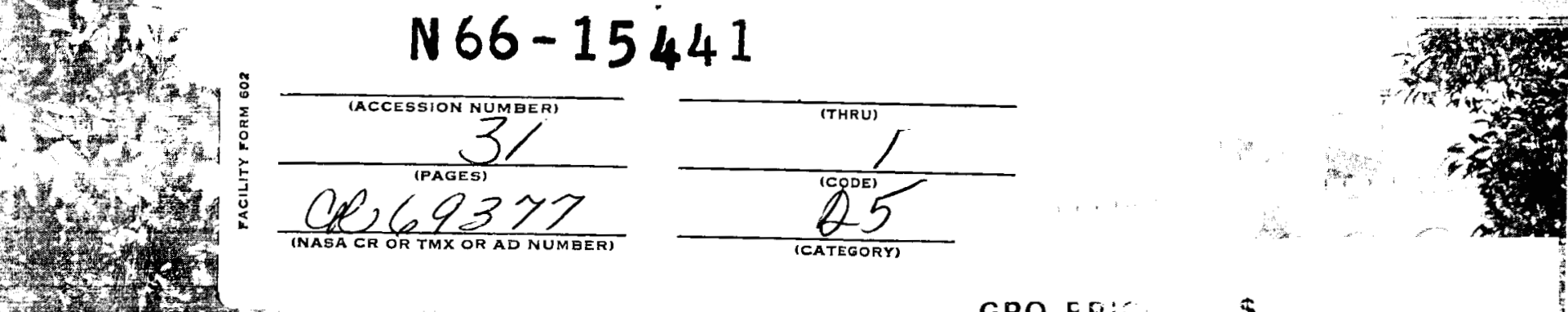

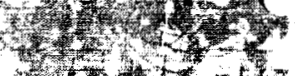

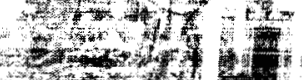

\title{
CFSTI Patis
}

$\$$

CFSTI PSTEAS

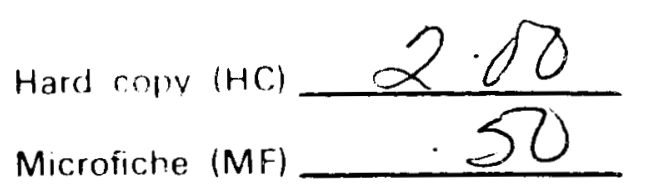

Microfiche (MF) 
Stability Limits for Longitudinal Waves

In Ion Beam-P lasma Interaction

Burton D. Fried and Alfred Y. Wong

August 1965

$R-2$

Plasma Physics Group
Department of Physics
University of California
Los Angeles, California 
This research was partially supported by the National Aeronautics and Space Administration under grant NGR-05-007-066. 


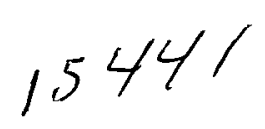

The two stream instability is examined for the case of an ion beam traversing a plasma. The dispersion equation for linearized, longitudinal waves in a plasma where collisions are negligible is used to find the restrictions on beam velocity, temperature and density which will lead to growing waves. 


\section{INTRODUCTION}

The two stream instability phenomenon, for longitudinal waves, was first discussed by Peirce ${ }^{l}$ for the case of an electron beam moving through a background of cold ions. Subsequently, Boyd, Gould and Field ${ }^{2}$ carried out experiments on this instability for the case of electrons traversing a neutral plasma, explaining their observations in terms of the interaction between the streaming electrons and the plasma electrons. The importance of the Peirce instability for a current-carrying plasma, in which, again, electrons stream past ions, was pointed out by Buneman, ${ }^{3}$ and the effects of non-zero plasma temperature in this case were studied by Jackson, by Bernstein and Kulsrud ${ }^{5}$ and by Fried and Gould. ${ }^{6}$ The current availability of quiescent cesium plasmas ${ }^{7}$ and of ion beam sources, ${ }^{8}$ the latter developed in conjunction with ion propulsion studies, makes quite practical the experimental investigation of this instability in a plasma traversed by an ion beam.

In contrast to the othe $r$ configurations, this one involves principally ion dynamics; the phase velocity of the waves in question is, typically, so much smaller than that of the electrons that the latter can be treated as a neutralizing negative fluid. Thus, it represents the opposite extreme from the experiment of Boyd, Gould and Field, where the electron dynamics must be treated in detail while that of the ions can be ignored. In contrast to both of these situations, the Buneman case requires correct treatment of the dynamics of both species. In a sense, however, the ion beam problem is more complicated than either of the other two cases, since here the dynamics of three "species" of particles enter (plasma ions, beam ions, and 
electrons) whereas the others involve only two species, (beam electrons and plasma electrons in the one case, plasma electrons and ions in the other).

The well known dispersion relation for linearized longitudinal waves relates the phase velocity, $u=\omega / k$, and wave number, $k$, to the parameters of the problem: densities, temperatures and beam velocity. Unfortunately, the (numerical) investigation of these relations is one of that large class of tasks which are trivial in principal but not in practice, owing to the multiplicity of parameters and variables involved and to the unpleasant nature of the functions (error functions of complex argument). Thus, after defining the parameters and discussing the dispersion equation in Section II, we indicate briefly, in Section III, the methods used in finding the stability limits. The results, in graphical form are given in Figs. 3 through 5 ; rough analytic approximations to these curves, derived from the use of asymptotic expressions, are discussed in Section IV. Constraints imposed by ion beam dynamics are examined briefly in Section $V$, and the results are expressed, in Fig. 6, in a form convenient for comparison with experiment. 


\section{THE DISPERSION EQUATION}

The dielectric constant for longitudinal waves in a multi-species plasma in which collisions can be neglected is given by ${ }^{9}$

$$
\varepsilon \cdot(\underline{k}, \omega)=1-\sum_{a}\left(\omega_{a}^{2} / k^{2}\right) \int d \underline{v} \underline{k} \cdot \nabla_{v} f_{a}(\underline{v})(\underline{k} \cdot \underline{v}-\omega)^{-1} g
$$

where species $a$ has distribution function $f_{a}(\underline{v})$ and plasma frequency $\omega_{a}=\left(4 \pi n_{a} q_{a} / m_{a}\right)^{1 / 2}$. This follows immediately from a linearized treatment of the response of the plasma to an external Iongitudinal electric field, $\underline{E}=-\nabla \phi$, using the Vlasov or correlationless kinetic equation to describe the plasma dynamics. The dispersion equation, giving, for example, the frequency, $\omega$, of waves of wave number $\underline{k}$, is simply

$$
\varepsilon(\underline{k}, \omega)=0
$$

Specializing to the case of Maxwellian distributions,

$$
f_{a}(\underline{v})=\pi^{-3 / 2} a_{a}^{-3} \exp \left[-\left(v-v_{a}\right)^{2} / a_{a}^{2}\right]
$$

we have the dispersion equation

$$
\varepsilon(\underline{k}, \omega)=1-\sum_{a}\left(k_{a}^{2} / 2 k^{2}\right) Z^{\prime} \cdot\left[\left(\omega / k-v_{a}\right) / a_{a}\right]=0
$$

where $Z^{\prime}$ is the derivative of the plasma dispersion function, 10

$$
z(s)=e^{-s^{2}}\left[i \pi^{1 / 2}-2 \int_{0}^{s} d t e^{t^{2}}\right] \text {, }
$$

and

$$
k_{a}^{2}=2 \omega_{a}^{2} / a_{a}^{2}=\text { Debye wavenumber for species } a .
$$


For the ion beam-plasma configuration, (4) becomes, in the plasma rest frame

$$
2 k^{2} / k_{i}^{2}=Z^{\prime}(u)+\left(n_{b} M_{i} a_{i}^{2} / n_{i} M_{b} a_{b}^{2}\right) Z^{\prime}\left[a_{i}(u-v) / a_{b}\right]+\left(n_{e} T_{i} / n_{i} T_{e}\right) Z^{\prime}\left(u a_{i} / a_{e}\right),
$$

where

$u=\omega / \mathbf{k}_{\mathbf{i}}$

$$
v=v_{b} / a_{i}
$$

are the dimensionless phase velocity and drift velocity, and the subscripts $i, b$, and e denote plasma ions, beam ions, and electrons, respectively. Both beam and plasma ions have been assumed univalent. It is convenient to choose units in which $a_{i}, k_{i}$, and $n_{i}$ are unity, and to define

$$
n=n_{b} / n_{i} \quad a=a_{i} / a_{b} \quad T=T_{i} / T_{e} .
$$

Specializing also to the case where the ions of the beam and plasma have equal mass, we have finally

$$
2 k^{2} / k_{i}^{2}=D(u) \equiv Z^{\prime}(u)+n a^{2} Z^{\prime}[a(u-V)]-2(n+1) T
$$

Here we have also assumed the phase velocity to be small compared to a and therefore have set $Z^{\prime}\left(\omega / k_{e_{e}}\right) \approx z^{\prime}(0)=-2$.

For given $k$ and given values of $n, a, V$, and $T,(8)$ can be solved, in principle, to give the phase velocity, $u$. In absence of a beam ( $n=0$ or $V=0$ ), all solutions of (8) have $\operatorname{Imu}<0$, corresponding to Landau-damped waves. For $n \neq 0$, there is a critical value of $V$ for which (8) first has a solution with real $u$; for larger $v$, there are unstable solutions, with Imu $>0$. Thus, to find the stability limit in the $n, a, V$ parameter space we look for solutinga of (8) with u rea.. f. convenient way of doing this is 
to use an on-line computing facility ${ }^{11}$ which includes graphical displays. By varying the parameters and observing the mapping of the complex $u$ plane generated by $D(u)$, it is easy to see when $D(u)=0$ has real roots, and to determine the parameter values to any desired accuracy. 


\section{DETERMINATION OF STABILITY LIMITS}

Consider the mapping, $w=D(u)$, from the $u$ plane to the $w$ plane, with D defined by (8). For a stable case, e.g. V =0, the curve, C, which is the map of the real $u$ axis, is as shown in Fig. 1a. It is clear that (8) has no solution with real $k$ and real $u$; since the upper half of the $\mathrm{u}$ plane maps into the interior of the closed curve $\mathrm{C}$, it is also clear that there are no growing waves. With fixed $n, a, T$ and increasing $V, C$ evolves as shown in Fig. Ib until, for a critical value, $V=V_{1}$, its intersection with the real w axis reaches the origin. $v_{1}$ is the minimum value of $V$, for this $n, T$ and $a$, for which the waves are unstable. Since both ReD and $\operatorname{ImD}$ vanish, the instability occurs first at $k=0$. As $V$ increases further, the origin in the w plane becomes, and remains, an interior point of the curve $C$, as shown in Fig. Ic until a second critical value $V=V_{2}$, is reached, when $C$ again passes through the origin. For $V>V_{2}$, the curve $C$ no longer encircles the origin (Fig. ld). Thus, the waves are unstable for $\mathrm{V}_{1}<\mathrm{V}<\mathrm{V}_{2}$ and are stable otherwise.

Once this global character of the stability problem is established, we can determine the stability limits with any desired accuracy by simply examining the map, not of the whole real $u$ axis, but only of that part which maps into the vicinity of the origin, as illustrated in Fig. 2. Carrying out this procedure keeping three of the four parameters $(n, a, V, T)$ fixed and varying the fourth gives contour lines for the stability limits such as those shown in Figs. 3 and 4 . The value of $u$ which maps into $w=0$ in a case like the middle curve of Fig. 2 is easily found; the result is shown in Fig. 5. 

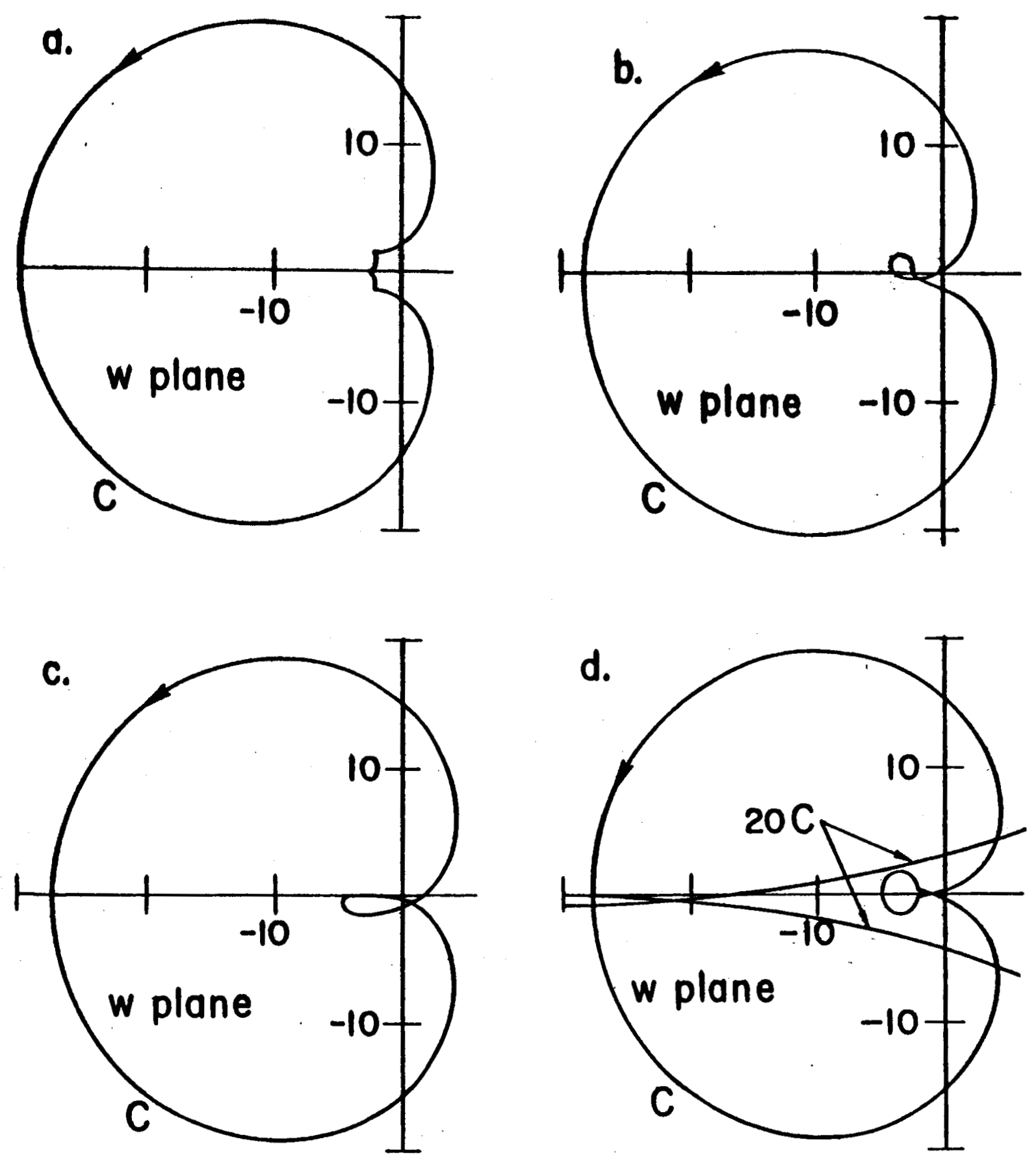

Fig. 1. Mapping, in the $w$ plane, of the real $u$ axis under the transformation $w=D(u)$, given by Eq. (8). As $u$ traverses the real axis, from left to right, $w$ traverses the curve $C$ in the direction shown by the arrow. All plots are for the case $n=0.5, a=5, T=1$, but with various values of the ion beam velocity, $V$, as follows: a) $V=0$, i. e., no beam is present. b) $V=0.7$. The curve $C$ does not encircle the origin since the beam is not yet fast enough to be unstable. c) $\mathrm{V}=1.75$. The curve $C$ now encircles the origin, showing that $V$ exceeds the threshold for instability. d) $V=3.0$. It is hard to tell whether or not $C$ encircles the origin, but by mapping a restricted (suitably chosen) portion of the $u$-axis we get, in effect, a magnified version (enlarged by a factor of 20) of the portions of $C$ in the vicinity of the origin, from which it is clear that for this value of $V$ the beam is stable. 


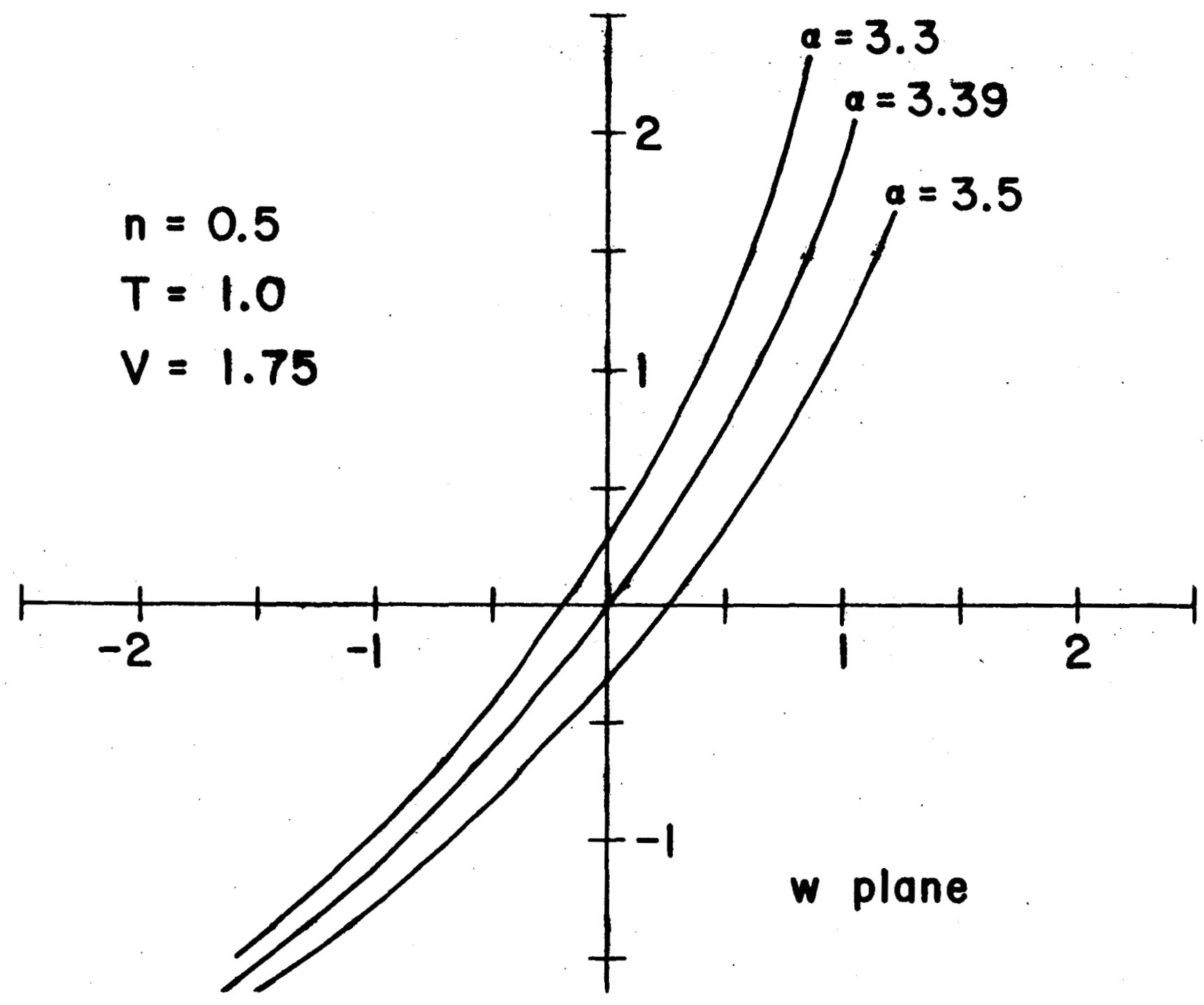

Fig. 2. Mapping of a restricted portion of the real u axis, $1 \leqslant u \leqslant 1.3$ showing how a precise root of the dispersion equation is determined. Here $\mathrm{n}=0.5, \mathrm{~T}=1.0$ and $\mathrm{V}=1.75$ are fixed and $a$ is varied. For $a=3.3$ it is clear that we have a stable configuration and that a must be increased to reach the stability limit. The value $a=3.5$ is too large, but interpolation on the intercepts of these curves with the $\mathrm{x}$ or $y$ axes leads to $a=3.39$, which proves to be correct to three significant figures. By mapping a smaller portion of the u-axis, we could, of course, determine a with any desired precision. 


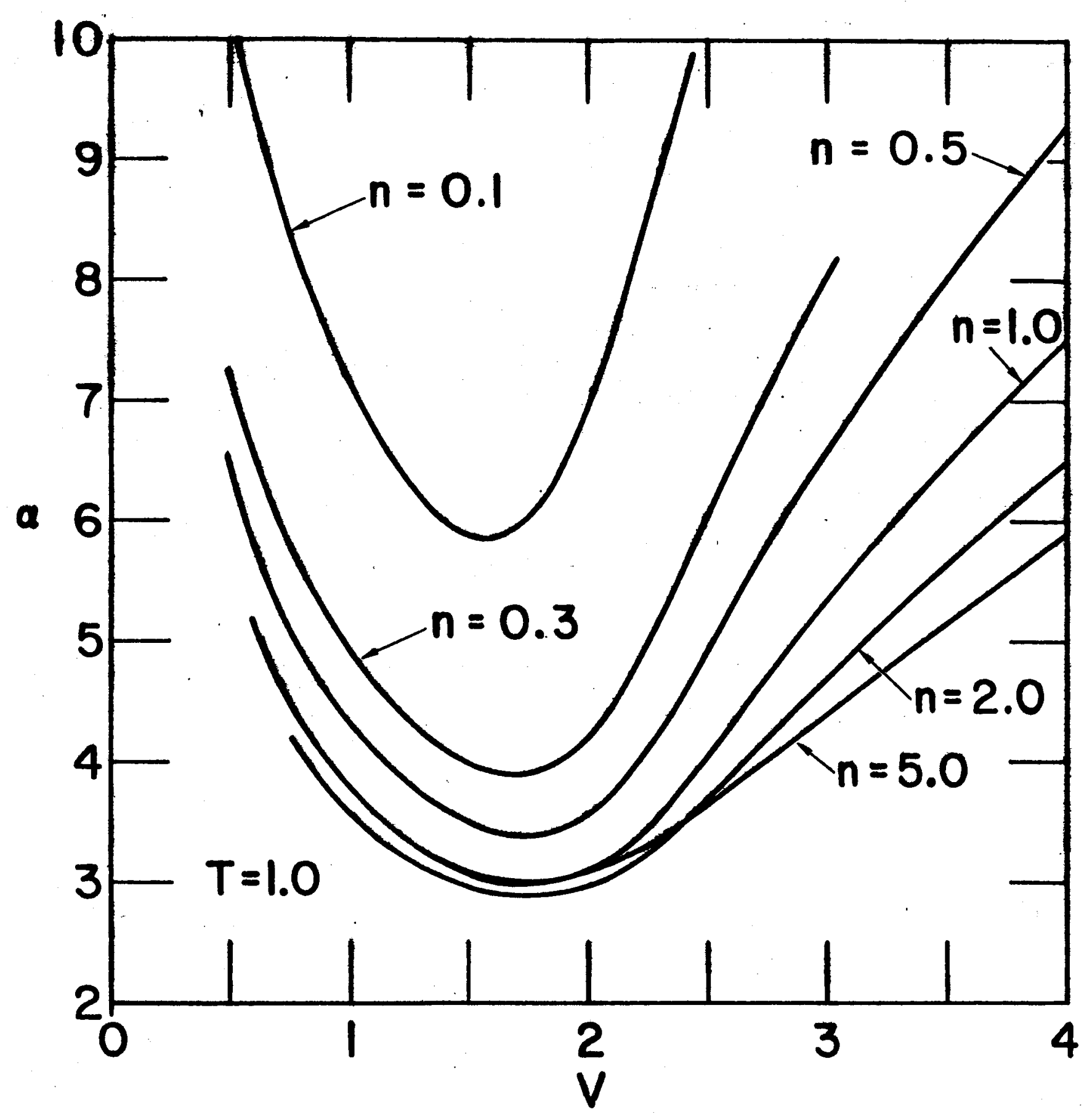

Fig. 3. Stability limit curves for a plasma with equal electron and ion temperatures, $T=T_{i} / T_{e}=1 . V$ is the ratio of ion beam mean velocity to $a_{i}$, the plasma ion thermal velocity, $a$ is the ratio of $a_{i}$ to the beam thermal velocity, $a=a_{i} / a_{b} \cdot n$ is the ratio of ion beam density to ion plasma density. For given $n$, the region below the curve corresponds to stability, and the region above the curve corresponds to growing waves. 


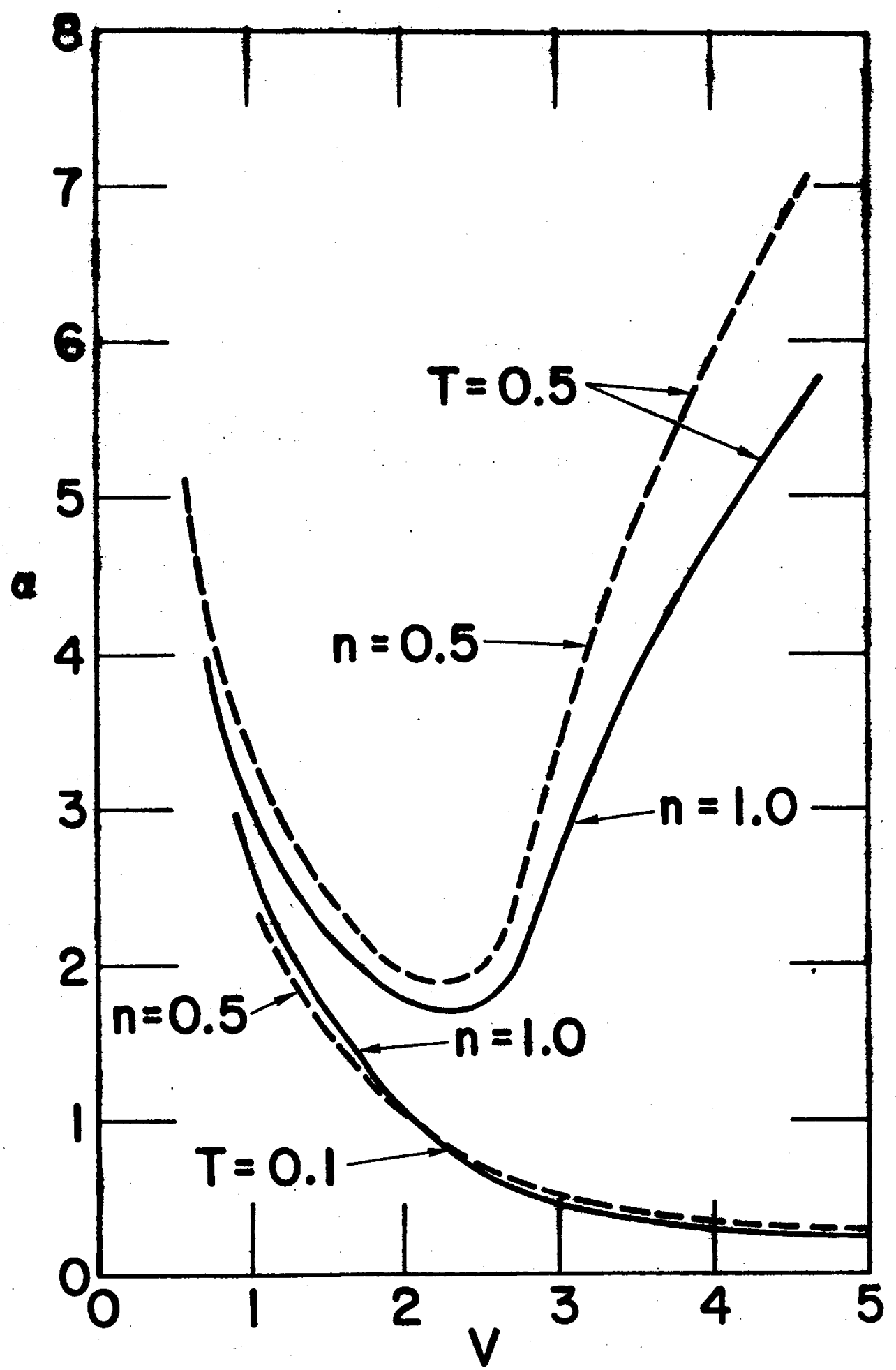

Fig. 4. Stability limit curves for $T=0.5$ and 0.1 and $n=0.5$ and 1.0 . 


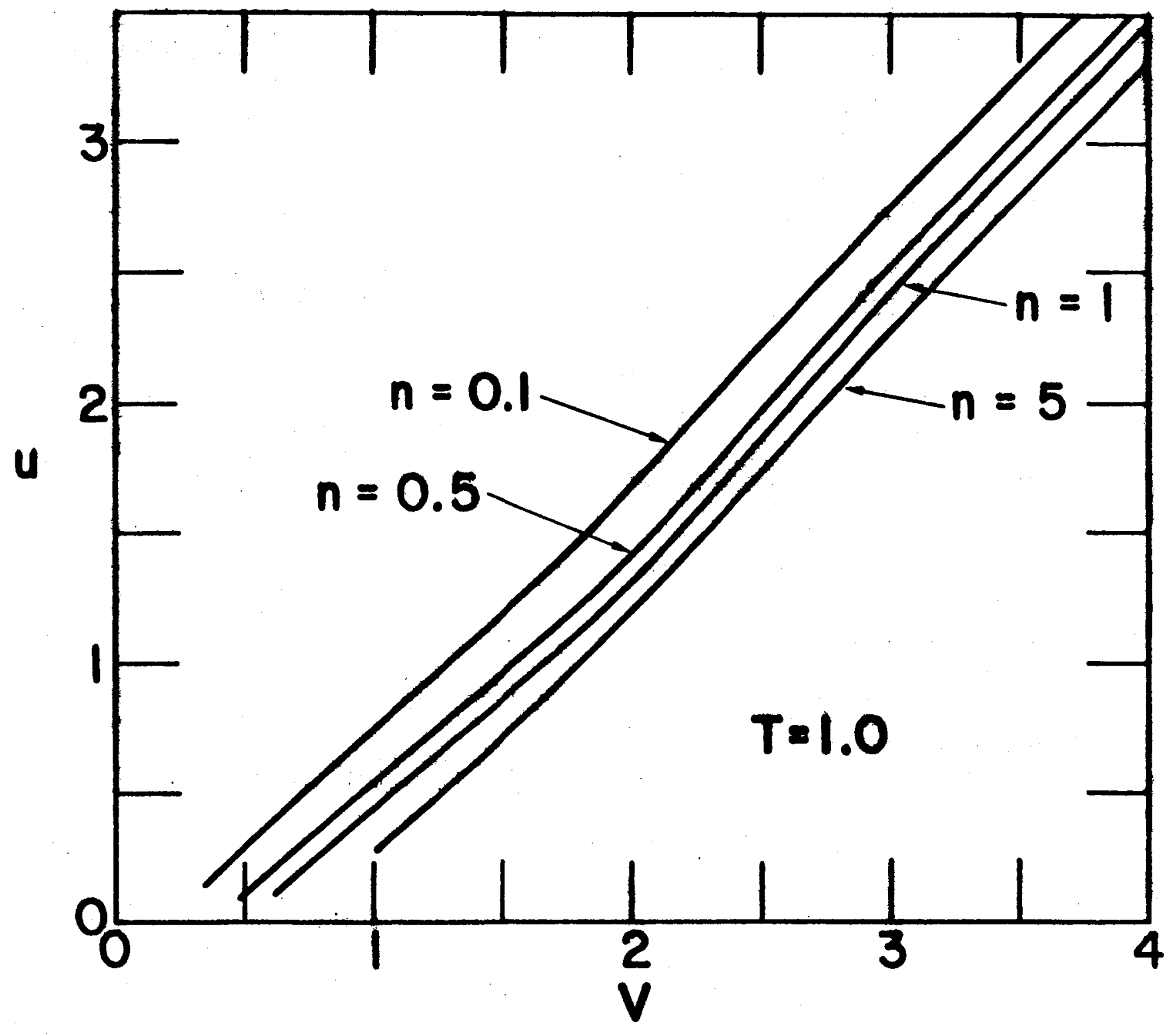

Fig. 5. Phase velocity, u, vs mean ion beam velocity for a marginally stable configuration with $T=1$ and several values of $n$. 
Although this point-by-point procedure can always be used when necessary, it is more convenient to determine one of the curves in Figs. 3 through 5 in a single process when possible. To do this, we set

$$
Z^{\prime}(u)=R(u)+i I(u)
$$

and rewrite (8) as the two equations

$$
\begin{gathered}
B(u) \equiv \frac{2(n+1) T-R(u)}{I(u)}=\frac{R[a(V-u)]}{I[a(V-u)]} \\
n a^{2}=I(u) / I[a(V-u)]
\end{gathered}
$$

The function

$$
L(x)=R(x) / I(x)
$$

is, for positive $x$, a monotone decreasing function of $x$, taking on all values, $+\infty>\mathrm{L}>-\infty$ as $\mathrm{x}$ varies from 0 to $\infty$. Thus, we can
1) Choose u.
2) Calculate B.
3) Use (10) to find $x=a(V-u)$.
4) Use (11) to calculate a.
5) Determine $v=x / a+u$.

Doing this simultaneously with a number of values of $u$ gives, directly, $a(u)$ and $V(u)$, and hence $a(V)$ and $u(V)$.

In deriving (8), we assumed $T=T_{i} / T_{e}$ fixed. In some cases, this probably corresponds to the experimental situation, e.g., a quiescent 
cesium plasma where the ion and electron temperatures are determined by the hot tungsten plate which generates the plasma so that $T_{i} / T_{e}=1$, while the ions of the injected beam have their own temperature $T_{b} \neq T_{i} A$ different possibility is to assume that $T_{e}$ is a weighted mean,

$$
n_{e} T_{e}=n_{b} T_{b}+n_{i} T_{i}=n_{i} T_{i}\left(1+n / a^{2}\right)
$$

so that

$$
\frac{T_{i}}{T_{e}}=\frac{(n+1)}{1+n / a^{2}}
$$

and instead of (8) we have

$$
2 k^{2}=\bar{D}(u) \equiv Z^{\prime}(u)+n a^{2} Z^{\prime}[a(u-V)]-2(n+1)^{2}\left(1+n / a^{2}\right)^{-1}
$$

The resultant stability limit curves are similar to those shown in Figs. 3 and 4, differing from these chiefly in having a second branch at large $\mathrm{V}$ and small a. Physically, this corresponds to the fact that (13), unlike (8), is completely symmetric under the interchange of beam ions and plasma ions, so that either of the two ion species may be considered as the "beam". Algebraically, this leads to the following property of (13): If (n, a, $v, u)$ is a solution of (13), so is $\left(n^{\prime}, a^{\prime}, V^{\prime}, u^{\prime}\right)$ with

$$
n^{\prime}=1 / n \quad a^{\prime}=1 / a \quad V^{\prime}=a V \quad u^{\prime}=a(u-V) .
$$




\section{ASYMPTOTIC FORMS}

For very large V, we see from Figs. 3 and 5 that $a$ and $u$ are also large. This suggests using asymptotic expansions for $\operatorname{Re} Z$ in (8):

$$
D(u) \approx \frac{1}{u^{2}}+\frac{n}{(u-V)^{2}}-2(n+1) T=0
$$

If $[u-V]<<u$, this gives

$$
V-u=\sqrt{\frac{1}{2} n /(n+1) T} \equiv g
$$

The imaginary part of $(8)$ is then

$$
\begin{aligned}
& u e^{-u^{2}}=n a^{3} g e^{-a^{2} g^{2}} \\
& u^{2}=a^{2} g^{2}+\log \left(u / n a^{3} g\right) .
\end{aligned}
$$

To leading order,

$$
\mathrm{u}=\mathbf{a} \mathbf{g}
$$

and so

$$
V=(a+1) \sqrt{\frac{1}{2} n /(n+1) T}
$$

This provides a rough approximation to the right hand portions of the curves in Fig. 3.

For very small $V$, $a$ is again large, but $u$ is small, so we use an asymptotic approximation for $\operatorname{Re} Z^{\prime}[a(u-V)]$ but replace $Z^{\prime}(u)$ by $Z^{\prime}(0)=-2$. With

$$
\mathbf{x}=\mathrm{V}-\mathrm{u}
$$


this gives for the real part of (8)

$$
\begin{aligned}
& n / x^{2}-2(n+1) T=2 \\
& x=\sqrt{\frac{1}{2} n /[(n+1) T+1]} .
\end{aligned}
$$

In the equation $\operatorname{ImD}=0$ we let $\mathrm{e}^{-\mathrm{u}^{2}}=1$, giving

$$
u=n a^{3} \times e^{-a^{2} x^{2}}
$$

so

$$
v=u+x=x\left[1+n a^{3} e^{-a^{2} x^{2}}\right]
$$

This shows that each curve of $V$ vs a with $n$ fixed has an asymptote at $V=\sqrt{\frac{1}{2} n /(n+2)}$, which is also consistent with the curves in Fig. 3. 
V. ION BEAM DYNAMICS

We have expressed the results heretofore in terms of the parameters most convenient for the stability analysis. However, these are not necessarily suitable for analysis of experiments with a beam-plasma system. For example, if ions are accelerated by a grid system and sent into a quiescent plasma, it is difficult to vary $V$ holding a fixed, or vice versa, since the two are coupled by the dynamics of the acceleration process. Thus, given an initial velocity distribution for the beam ions and a net accelerating potential, $\phi$, we must solve for the average velocity of the accelerated ions and use this for the beam velocity, $V_{b}$, of Section II. It will differ somewhat from $\sqrt{2 e \phi / M}$, which will, in fact, be the minimum velocity of the beam ions. Likewise, we must use the thermal width of the accelerated beam as the $a_{b}$ of Section II; in general it will be smaller than that of the ion source. We discuss briefly an elementary example of these considerations.

At the ion beam source, the distribution function of beam ions is assumed to be a half-Maxwellian

$$
f_{0}(v)= \begin{cases}n \exp \left(-v^{2} / a_{0}^{2}\right) & v>0 \\ 0 & v<0\end{cases}
$$

since ions with $\mathrm{v}<0$ are readsorbed by the source. If the ions fall through a potential difference

$$
\Delta \phi=\mathrm{Mv}_{1}^{2} / 2 \mathrm{q}
$$

their distribution function will be, according to the time independent Vlasov 
equation,

$$
f_{1}(v)= \begin{cases}N \exp \left(\left[v_{1}^{2}-v^{2}\right] / a_{0}^{2}\right) & v>v_{1} \\ 0 & v<v_{1}\end{cases}
$$

from which it follows that their mean velocity is

$$
\langle v\rangle=\int d v v f_{1}(v) / \int d v f_{1}(v)=a_{0} E\left(v_{1} / a_{0}\right) \quad E(x) \equiv e^{-x^{2}} / \sqrt{\pi} \operatorname{erfc}(x)
$$

and that their mean thermal velocity is

$$
a_{b}=\sqrt{2}\left(\left\langle v^{2}\right\rangle-\langle v\rangle^{2}\right)^{1 / 2}=a_{0}\left[F\left(v_{1} / a_{0}\right)\right]^{1 / 2}
$$

where

$$
F(x)=1+2[x-E(x)] E(x)
$$

and

$$
\operatorname{erfc}(z)=(2 / \sqrt{\pi}) \int_{z}^{\infty} d t \exp \left(-t^{2}\right)
$$

That is to say, the Maxwellian "equivalent" to the beam is

$$
\exp \left[-(v-\langle v\rangle)^{2} / a_{b}^{2}\right] / \sqrt{\pi} a_{b}
$$

The parameters, $v=\langle v\rangle / a_{i}$ and $a=a_{i} / a_{b}$, of our stability analysis are therefore given by

$$
\begin{aligned}
& v=\left(a_{0} / a_{i}\right) E\left(v_{1} / a_{0}\right) \\
& a=\left(a_{i} / a_{0}\right)\left[F\left(v_{1} / a_{0}\right)\right]^{-1 / 2}
\end{aligned}
$$

In an experiment, the density ratio of beam to plasma, $n$, and the accelerating potential, $\Delta \phi$, can be easily varied, while $T_{i^{*}} T_{0^{\circ}}$ and $T_{e}$ are held fixed. It is therefore convenient to find the stability limits in an $n, \Delta \phi$ 
plane, which we do as follows. With $T_{i}, T_{0}$, and $\Delta \phi$ given, $v_{1}$ is known $\left(v_{1}=\sqrt{2 q \Delta \phi / M)}\right.$ and (17) determines $V$ and $a$. If $T_{e}$ is also given, then we can, as in Section III, determine the value of $\mathbf{n}$ corresponding to the stability limit. The resulting plot of $n$ vs $\left(q \Delta \phi / T_{i}\right)^{1 / 2}$ is shown in Fig. 6 , for several values of $a_{e} / a_{i}$ and $a_{i} / a_{0}$. 

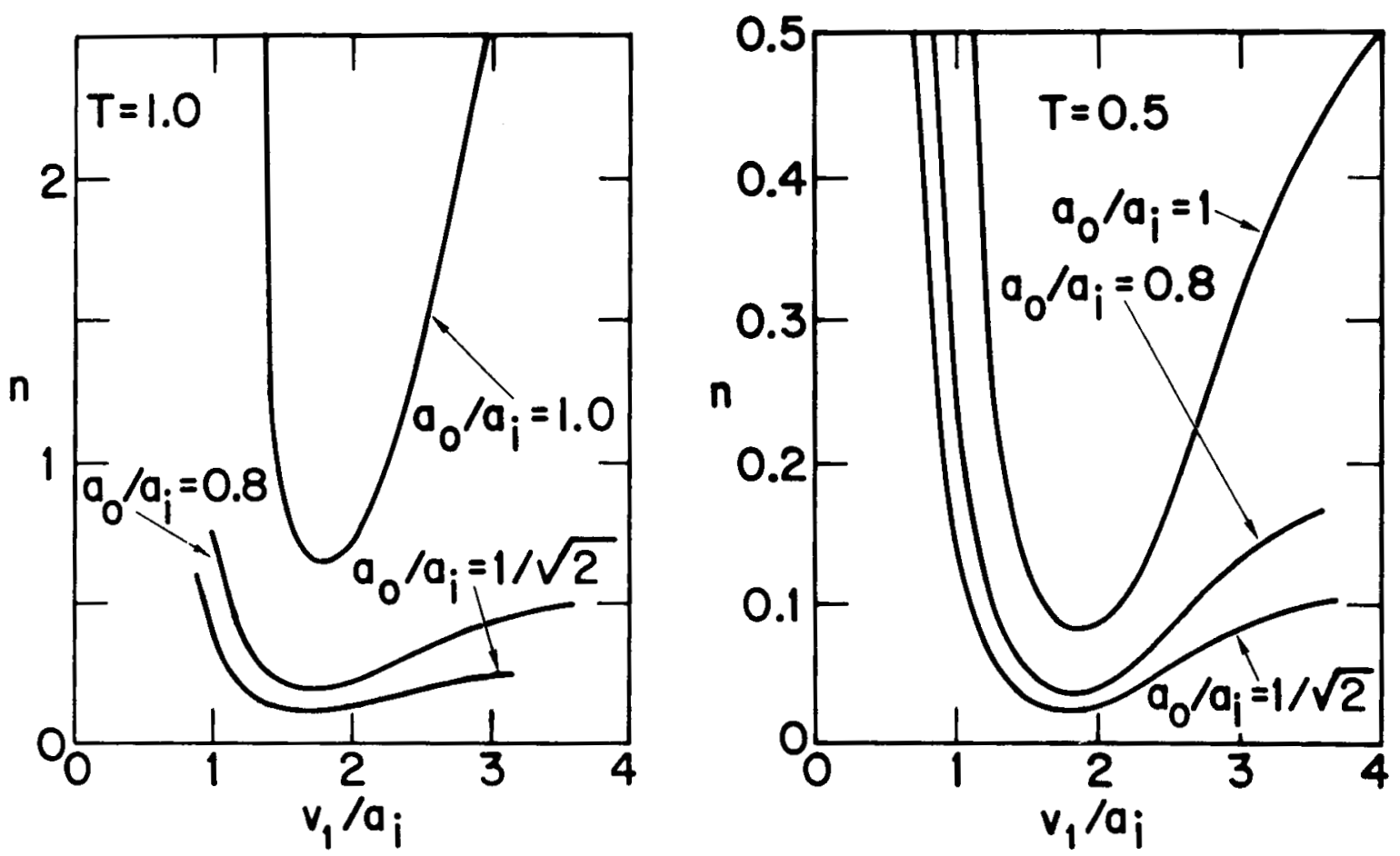

Fig. 6. Stability limit curves taking into account ion beam dynamics. The ordinate is $\mathrm{n}$, the ratio of ion beam density to plasma ion density. The absiccae is the ratio of minimum beam velocity to ion thermal velocity or, equivalently, $\sqrt{q \Delta} \overline{\phi / T}$, where $\Delta \phi$ is the net accelerating potential for the ion beam. Results for three values of the ratio $\left(a_{0} / a_{i}\right)^{2}=$ (ion source temperature/plasma ion temperature) and two values of the ratio $T=$ (plasma ion temperature/plasma electron temperature) are plotted. 


\section{CONCLUSIONS AND DISCUSSION OF RESULTS}

The stability limit curves shown in Figs. 3 and 4 are based on a collisionless plasma with Maxwellian distribution functions for both beam and plasma. They should be qualitatively correct for actual plasmas where the distribution functions are similar to Maxwellians and the collision frequencies are small compared to the wave frequency, $\omega$ : Although the curves apply strictly to the case $k=0$, they are valid for $k<<k_{i}=$ Debye wave number of the plasma ions. In a real plasma, the minimum wave number, $k_{\text {min' }}$ will be set by some physical consideration such as plasma dimensions. The wave frequency of the marginally unstable waves will then be given by $\omega=k_{\min } u$, where $u$, as shown in Fig. 5, is approximately proportional to the drift velocity.

From Figs. 3 and 4 we see that for given $n$ there is a minimum, $a_{\text {min' }}$ (and hence a maximum beam velocity spread) in order that any wave be unstable. For smaller $a$, the Landau damping is apparently larger than the growth due to the two stream instability. Moreover, for a $>a_{\text {min' }}$ the beam velocity must be neither too large nor too small if the waves are to be unstable. The fact that the curves have a shape suggesting a broad resonance, centered between $V=1.5$ and 2 , may be attributed to the fact that in the equilibrium . plasma the ion acoustic waves have a phase velocity of the order of ion thermal velocity, and, for $T=1$, are strongly damped. ${ }^{6}$ (Thus, the least damped root of (8) for $n=V=k=0, T=1$ is at $u \approx 1.5-i 0.6$, so we expect the maximum interaction with the beam to occur at $\mathrm{V}$ of order 1.5 and to be quite broad.) For larger $T_{e} / T_{i}$, the ion acoustic waves are less damped 6 , which is 
reflected in the lower a threshold of Fig. 4 and the sharper nature of the resonance for $T=0.5$. (For $n=k=V=0$ and $T=0.5$, the least damped root of (8) is $u \approx 1.7-i 0.4 .1$

It should be emphasized that in a laboratory plasma, as opposed to our idealized, uniform, homogeneous model, other instabilities may be more important than the longitudinal modes considered here. For example, if the plasma is confined by an external magnetic field, as in the cesium plasma devices, instabilities associated with gradients of density are commonly observed. A separate study of each of these other possibly unstable modes is necessary in order to predict which will actualy dominate for any given choice of the parameters of the system.

We are indebted to A. Klimeck and J. Craft for assistance with the numerical calculations, most of which were done with the TRW On-Line Computer. This work was partially supported by the TRW Independent Research Program, the Office of Naval Research, and the National Aeronautics and Space Administration. 


\section{FIGURES}

1. Mapping, in the $w$ plane, of the real $u$ axis under the transformation $w=D(u)$, given by Eq. (8). As u traverses the real axis, from left to right, w traverses the curve $\mathrm{C}$ in the direction shown by the arrow. All plots are for the case $n=0.5, a=5, T=1$, but with various values of the ion beam velocity, $V$, as follows: a) $V=0$, i.e., no beam is present. b) $V=0.7$. The curve $C$ does not encircle the origin since the beam is not yet fast enough to be unstable. c) $V=1.75$. The curve $C$ now encircles the origin, showing that $V$ exceeds the threshold for instability. d) $V=3.0$. It is hard to tell whether or not $C$ encricles the origin, but by mapping a restricted (suitably chosen) portion of the u-axis we get, in effect, a magnified version (enlarged by a factor 20) of the portions of $\mathrm{C}$ in the vicinity of the origin, from which it is clear that for this value of $V$ the beam is stable.

2. Mapping of a restricted portion of the real $u$ axis, $1 \leqslant u \leqslant 1.3$ showing how a precise root of the dispersion equation is determined. Here $\mathrm{n}=0.5, \mathrm{~T}=1.0$ and $\mathrm{V}=1.75$ are fixed and $\mathrm{a}$ is varied. For $a=3.3$ it is clear that we have a stable configuration and that a must be increased to reach the stability limit. The value $a=3.5$ is too large, but interpolation on the intercepts of these curves with the $x$ or $y$ axes leads to $a=3.39$, which proves to be correct to three significant figures. By mapping a smaller portion of the u-axis, we could, of course, determine a with any desired precision. 


\section{FIGURES}

(cont'd)

3. Stability limit curves for a plasma with equal electron and ion temperatures, $T=T_{i} / T_{e}=1 . V$ is the ratio of ion beam mean velocity to $a_{i}$, the plasma ion thermal velocity. $a$ is the ratio of $a_{i}$ to the beam thermal velocity, $a=a_{i} / a_{b}, n$ is the ratio of ion beam density to ion plasma density. For given $n$, the region below the curve corresponds to stability, and the region above the curve corresponds to growing waves.

4. Stability limit curves for $\mathrm{T}=0.5$, and 0.1 and $\mathrm{n}=0.5$ and 1.0 .

5. Phase velocity, $u$, vs mean ion beam velocity for a marginally stable configuration with $T=1$ and several values of $n$.

6. Stability limit curves taking into account ion beam dynamics. The ordinate is $n$, the ratio of ion beam density to plasma ion density. The absiccae is the ratio of minimum beam velocity to ion thermal velocity or, equivalently, $\sqrt{\mathrm{q}_{\Delta \phi} / \mathrm{T}_{i}}$, where $\Delta \phi$ is the net accelerating potential for the ion beam. Results for three values of the ratio $\left(a_{0} / a_{i}\right)^{2}=$ (ion source temperature/plasma ion temperature) and two values of the ratio $T=$ (plasma ion temperature/plasma electron temperature) are plotted. 


\section{REFERENCES}

${ }^{1}$ J. R. Peirce, J. App. Phys. 19231 (1948).

${ }^{2}$ G. D. Boyd, R. W. Gould, and L. M. Field, Proc. I. R.E. 491906 (1961).

${ }^{3}$ O. Buneman, Phys. Rev. Letters 118 (1958).

${ }^{4}$ J. D. Jackson, J. Nucl. Energy: Pt. C 1171 (1960).

${ }^{5}$ I. Bernstein and R. Kulsrud, Phys. Fluids $\underline{3} 937$ (1960).

${ }^{6}$ B. D. Fried and R. W. Gould, Phys. Fluids $\underline{4} 139$ (1961).

${ }^{7}$ See, for instance, N. Rynn and N. D'Angelo, Rev. Sci. Instr. 311326 (1960).

${ }^{8}$ See, for instance, J. M. Sellen, Rev. Sci, Instr. 36316 (1965).

${ }^{9}$ D. Montgomery and D. A. Tidman, Plasma Kinetic Theory. McGraw Hill New York (1964).

${ }^{10}$ B. D. Fried and S. Conte, The Plasma Dispersion Function, Academic Press, New York (1961).

${ }^{11}$ B. D. Fried, "STL On-Line Computer," TRW Systems Report 9824-6001-RU000 (1964). 